\title{
The Conundrum of Treating Portal Vein Thrombosis and Submucosal Esophageal Hematoma
}

\author{
Davinder Singh ${ }^{1}$, Kamran Zaheer ${ }^{1}$, Varun Dobariya ${ }^{1}$, Alexis P. Lester ${ }^{1}$, Samson Teka ${ }^{1}$ \\ 1. Internal Medicine, Joan C. Edwards School of Medicine - Marshall University, Huntington, USA
}

Corresponding author: Davinder Singh, singhda@marshall.edu

\begin{abstract}
Submucosal esophageal hematoma (SEH) is an uncommon clinical entity and a rare form of esophageal insult. Patients usually present with retrosternal chest pain and dysphagia, which often make the diagnosis of SEH difficult as it mimics common cardiovascular and pulmonary disorders. One of the common inciting factors includes the use of anticoagulants. In this report, we discuss the case of a patient with portal vein thrombosis who was treated with heparin and consequently developed SEH.
\end{abstract}

Categories: Internal Medicine, Medical Education, Gastroenterology

Keywords: submucosal, hematoma, esophageal, anticoagulant, heparin drip, portal vein thrombosis

\section{Introduction}

Hematomas of the esophagus are a rare part of a spectrum of injuries. Some of the common esophageal complications are tears (Mallory-Weiss syndrome) and esophageal ruptures (Boerhaave syndrome) [1]. Making the correct diagnosis in these patients is very important as symptoms may mimic other common differentials such as myocardial infarctions or pulmonary thromboembolism. Other differential diagnoses include spontaneous rupture of the stomach, acute pericarditis, pneumothorax, dissecting aneurysms, and pancreatitis [2]. Recognizing clinical signs early can not only help with narrowing the diagnosis but also prevents worsening complications such as bleeding and perforation. The classical triad of symptoms includes hematemesis, chest pain, and sudden dysphagia or odynophagia [3]. We discuss a patient who presented with two of the three classical symptoms. With the increasing use of multiple antiplatelet agents and the increased risk for esophageal hematoma formation, clinicians should be aware of the condition and avoid misdiagnosis. More specifically, patients with cirrhosis complicated with portal vein thrombus are placed on anticoagulation to prevent clot extension. In this report, we present a difficult case where submucosal esophageal hematoma (SEH) was a byproduct of treating portal vein thrombus with heparin.

Review began 10/28/2020 Review ended 12/01/2020 Published 12/11/2020

\section{(c) Copyright 2020}

Singh et al. This is an open access article distributed under the terms of the Creative Commons Attribution License CC-BY 4.0., which permits unrestricted use, distribution, and reproduction in any medium, provided the original author and source are credited.

\section{Case Presentation}

A 42-year-old female presented to the hospital with increased abdominal swelling and bilateral leg swelling for one month. The patient's past medical history was significant for cirrhosis secondary to alcohol abuse, intravenous drug use (IVDU), hepatitis C, hypersensitivity lung disease (HLD), and pancreatitis. Initial labs were remarkable for elevated prothrombin time, international normalized ratio (INR), total bilirubin, leukocytosis, and lactic acid. She was started on piperacillin-tazobactam (Zosyn $\left.{ }^{\circledR}\right)$ for coverage of possible spontaneous bacterial peritonitis. Initial CT imaging revealed portal vein thrombosis (Figure 1) and the patient was consequently started on a heparin drip. The following day, the patient reported melena and episodes of hematemesis, and heparin was discontinued. Next, the patient was transfused with 2 units of packed red blood cells and started on octreotide and pantoprazole drip. Her hemoglobin improved from 6.6 to 7.4. Due to the ongoing bleeding, the patient underwent an endoscopy, which revealed SEH without variceal bleeding (Figure 2). 


\section{Cureus}

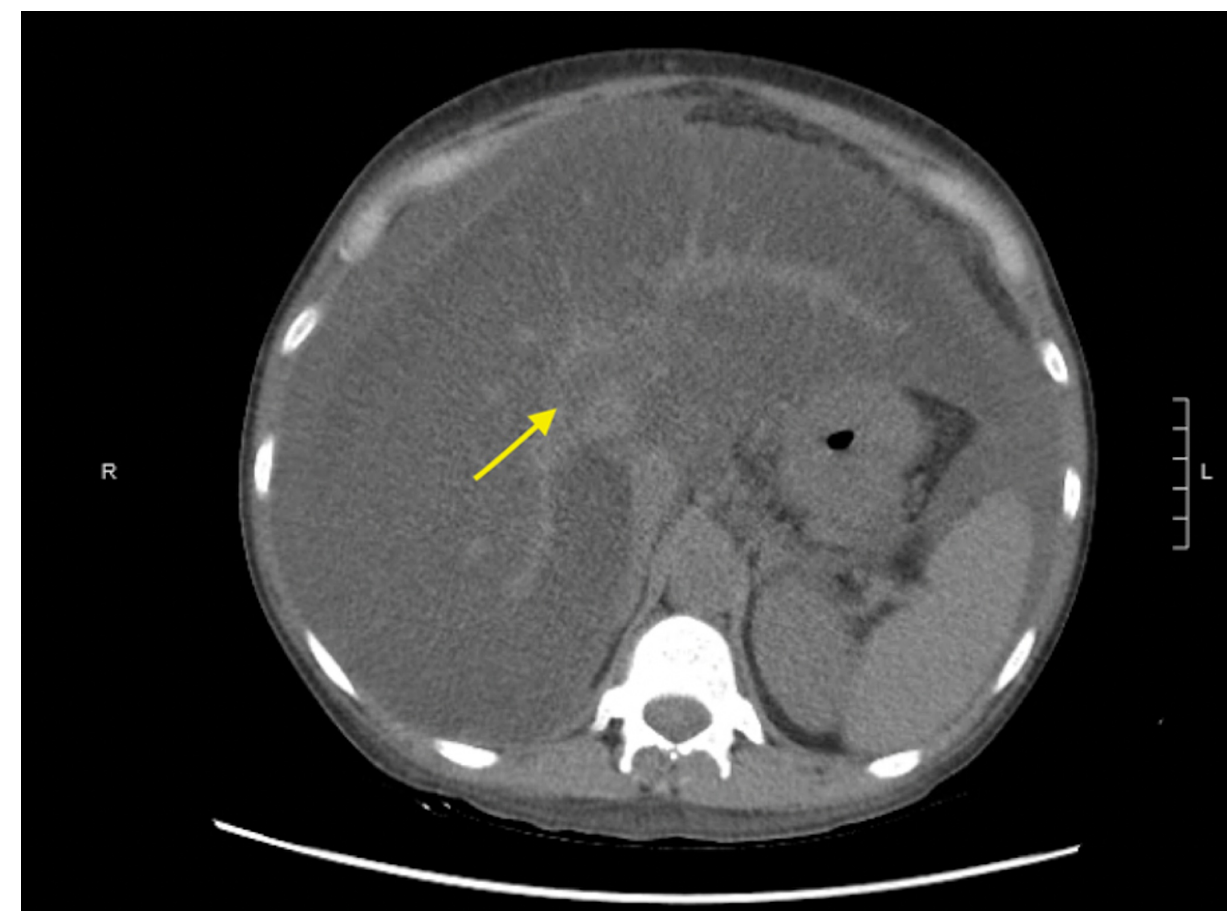

FIGURE 1: CT of the abdomen without contrast displaying portal vein thrombosis (arrow)

CT: computed tomography

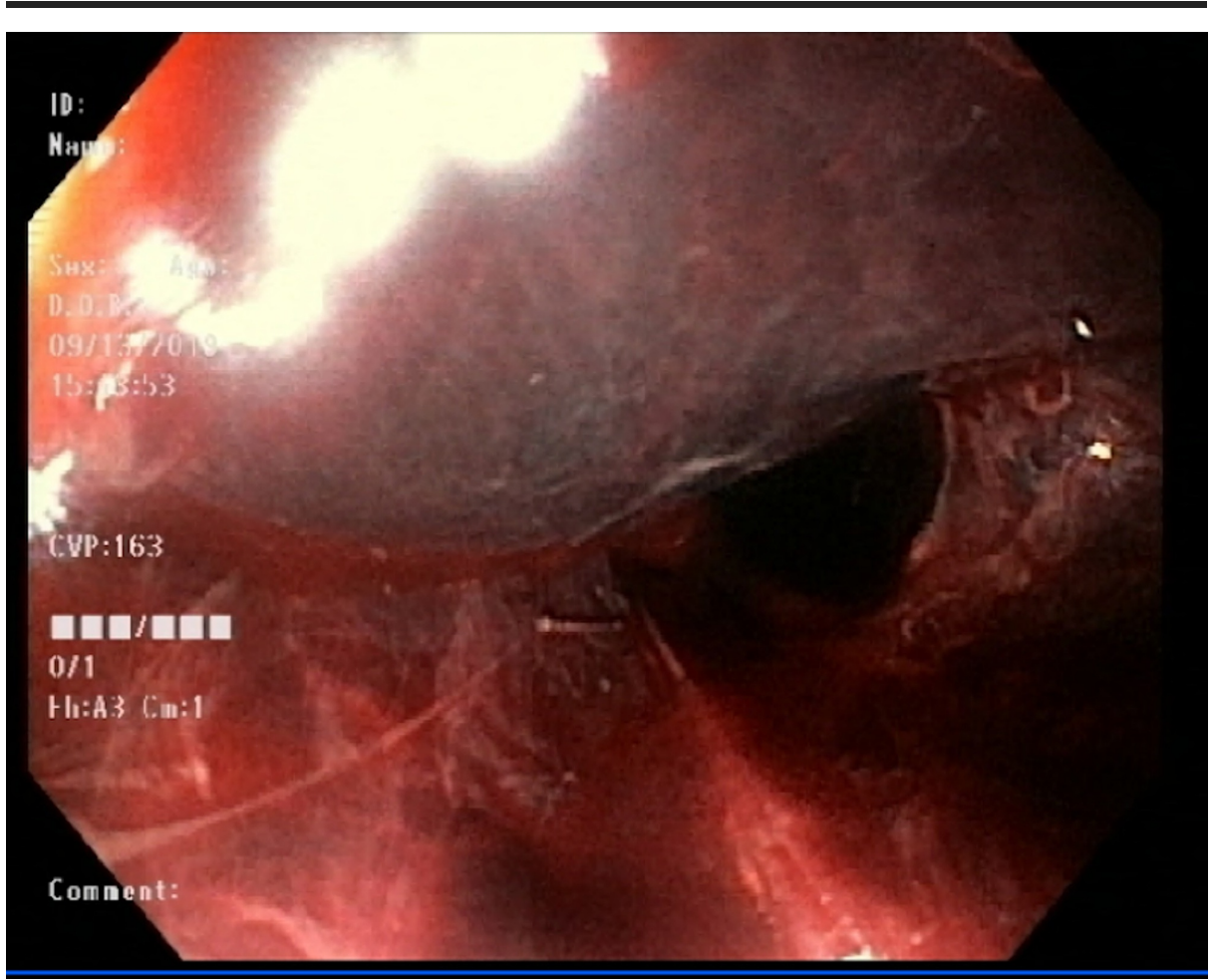

FIGURE 2: Esophagogastroduodenoscopy showing esophageal submucosal hematoma

The patient's hemoglobin on admission was 13.6, and on the day of episodes of hematemesis, it was 7.4. The gastroenterologist placed a nasogastric (NG) tube for nutrition via endoscopy. Following the above

measures, the patient became hemodynamically stable and the bleeding was stopped. Repeat abdominal CT 


\section{Cureus}

after conservative measures showed no signs of bleeding in the esophagus (Figure 3). Repeat abdominal ultrasound showed no indications of thrombosis and displayed a patent portal vein with bidirectional blood flow (Figure 4).

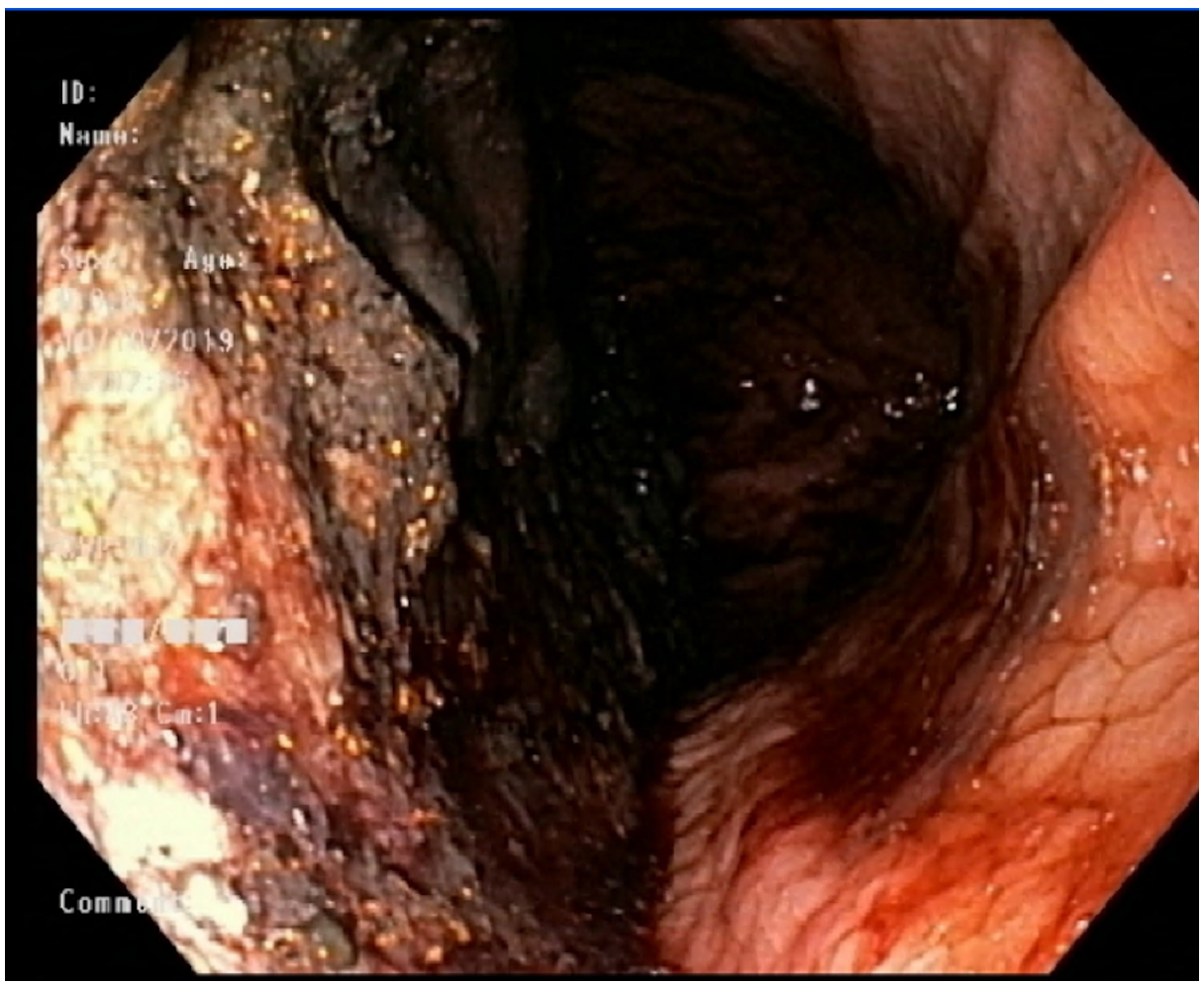

FIGURE 3: Repeat esophagogastroduodenoscopy showing no bleeding in the esophagus

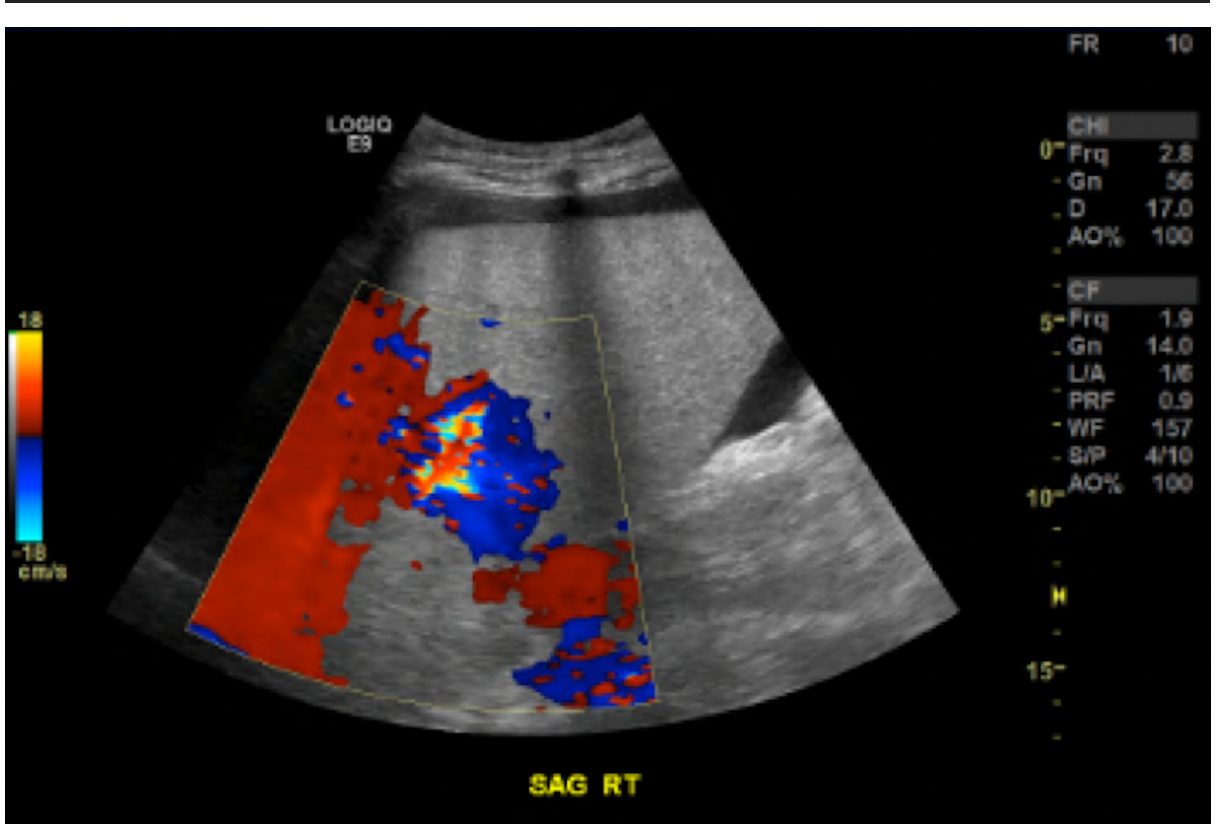

FIGURE 4: Bidirectional blood flow in the portal vein with no indication of thrombosis

\section{Discussion}

A hematoma of the esophagus is a rare condition characterized by a collection of blood in the wall of the 
esophagus. Clinical signs of SEH are unreliable and hence require further investigation. CT scans can reveal the intramural soft tissue density seen in hematomas [4]. Other imaging modalities such as esophageal swallowing study or upper gastrointestinal (GI) endoscopy are used for further confirmation of SEH or for inconclusive CT results. SEH can be further classified into four stages where stage I represents an isolated hematoma and stage II is characterized by hematoma being surrounded by tissue edema. In stage III, the hematoma with edema physically compresses the esophageal lumen, which causes respiratory difficulty leading to intubation. Stage IV is defined by the hematoma obliterating the esophageal lumen [4]. Our patient had stage III SEH, and due to early detection, she was managed conservatively and discharged home safely.

Classical clinical symptoms of SEH consist of retrosternal pain, dysphagia, and upper GI bleeding. It is believed that excessive anticoagulation and mechanical forces of retching account for the majority of the cases [5]. Our patient was on a heparin drip and had hematemesis early during her hospital course. The use of anticoagulants or antiplatelet therapy can cause hematomas in all parts of the GI tract including the esophagus, stomach, duodenum, and cecum [4]. Even though hematomas are considered benign, correct diagnosis is important since the symptoms mimic other common chest pain pathologies. Endoscopic evaluation can provide a reliable method for diagnosing hematomas since they have a distinctive endoscopic appearance. Submucosal hematomas involve the distal esophagus and often extend into the middle third and occasionally the upper third of the esophagus [6,7]. Another differentiating factor is the inclusion of the female sex. Esophageal hematomas predominantly affect females (80\%) while Mallory-Weiss syndrome (80\%) and Boerhaave syndrome (60-78\%) mainly affect males [8,9]. While Mallory-Weiss syndrome can also present with small submucosal hematomas, they often have a different location and characterization. The submucosal hematomas seen in Mallory-Weiss are localized and do not spread beyond 1-2 cm, which is explained by the mucosal tear decompressing the hematoma [10].

Misdiagnosis can result in excessive use of anticoagulant therapy resulting in worsening of bleeding. Our patient initially presented with abdominal swelling and pain, and after a proper diagnostic workup, she was placed on heparin for portal vein thrombosis. The anticoagulant may have caused active bleeding leading to hematemesis and melaena and further complicated her condition. We determined that the patient had SEH only after she was endoscopically examined. The patient was kept on conservative treatment until her vitals stabilized and repeat imaging confirmed the resolution of SEH and patent portal vein.

\section{Conclusions}

While diagnosing esophageal hematomas can prove to be difficult, early recognition and detection can help improve hospital stay and prevent further complications. Cardiac and pulmonary causes of the chest and abdomen present difficulty in distinguishing GI symptoms. Various imaging modalities provide invaluable information on the location, size, and extent of the hematomas. An accurate diagnosis of the hematoma can help physicians avoid giving anticoagulants and performing unnecessary tests that can lead to perforation or bleeding. By providing patients with conservative treatment early in their hospital stay, surgery can be prevented.

\section{Additional Information \\ Disclosures}

Human subjects: Consent was obtained by all participants in this study. Conflicts of interest: In compliance with the ICMJE uniform disclosure form, all authors declare the following: Payment/services info: All authors have declared that no financial support was received from any organization for the submitted work. Financial relationships: All authors have declared that they have no financial relationships at present or within the previous three years with any organizations that might have an interest in the submitted work. Other relationships: All authors have declared that there are no other relationships or activities that could appear to have influenced the submitted work.

\section{References}

1. Cheung J, Müller N, Weiss A: Spontaneous intramural esophageal hematoma: case report and review . Can J Gastroenterol. 2006, 20:285-286. 10.1155/2006/764714

2. Atefi D, Horney JT, Eaton SB, Shulman M, Whaley W, Galambos JT: Spontaneous intramural of hematoma of esophagus. Gastrointest Endosc. 1978, 24:172-174. 10.1016/s0016-5107(78)73499-1

3. Cao DT, Reny JL, Lanthier N, Frossard JL: Intramural hematoma of the esophagus. Case Rep Gastroenterol. 2012, 6:510-517. 10.1159/000341808

4. Efe C, Ozseker B, Kav T, Harmanci O, Bayraktar Y: Intramural hematoma of the esophagus as a complication of anticoagulant or antiplatelet therapy. Scand J Gastroenterol. 2011, 46:1399-1400. $10.3109 / 00365521.2011 .591429$

5. Snyder N 3rd, Patterson M, Hughes WS: Esophageal hematoma. South Med J. 1973, 66:1079-1080. 10.1097/00007611-197309000-00031

6. Cullen SN, McIntyre AS: Dissecting intramural haematoma of the oesophagus . Eur J Gastroenterol Hepatol. 2000, 12:1151-1162. 10.1097/00042737-200012100-00014

7. Furukawa H, Hara T, Taniguchi T, Tetsu O: A case of spontaneous intramural hematoma of the esophagus . Gastroenterol Jpn. 1993, 28:81-87. 10.1007/BF02775007 


\section{Cureus}

8. Hess U, Brücher BL, Cavallaro A, Hannig C, Stein HJ, Ott R: Intramural esophageal hematoma after cardioversion. Dis Esophagus. 1997, 10:225-228. 10.1093/dote/10.3.225

9. Yamashita K, Okuda H, Fukushima H, Arimura Y, Endo T, Imai K: A case of intramural esophageal hematoma: complication of anticoagulation with heparin. Gastrointest Endosc. 2000, 52:559-561. 10.1067/mge.2000.108664

10. Spiller RC, Catto JV, Kane SP: Spontaneous dissecting intramural haematoma of the oesophagus: a rare cause of haematemesis and dysphagia. Endoscopy. 1981, 13:128-130. 10.1055/s-2007-1021665 